\title{
Sizing and siting of distributed generation in distribution systems for voltage improvement and loss reduction
}

\author{
Mohamed Shaaban", J. O. Petinrin \\ Centre of Electrical Energy System (CEES), Faculty of Electrical Engineering, Universiti Teknologi Malaysia \\ 81310 UTM Johor Bahru, Johor, Malaysia.e-mail:m.shaaban@fke.utm.my; jopetinrin2@live.utm.my
}

\begin{abstract}
The integration of distributed generation (DG) into the distribution system brings conspicuous technical and economic benefits to the grid. Yet installation of non optimized sizes of DGs at arbitrary locations can result in degraded voltage profile and increased system losses. This would consequently drive cost escalation, creating an effect against the desired. It is therefore expedient that the sizing and location of DG units should be prudently planned and determined. This paper presents an analytical technique to determine the optimal size and siting of DGs in a distribution system for active power loss reduction and voltage profile improvement. DG unit sizing and siting are calculated using a sensitivity index that combines the exact loss formula and voltage sensitivity coefficients. Investigation is carried out on the IEEE 13 bus test feeder to validate the effectiveness of the proposed method. Aspects related to the connection of non-dispatchable renewable DG to the distribution network and the use of energy storage, as a representative of smart grid technologies, is also discussed.
\end{abstract}

Keywords: Distribution network, distributed generation, optimum size, optimum location, power loss, voltage profile.

\section{Introduction}

The problem of global warming and exhaustion of fossil fuel have fostered the increased usage of less environmentally-polluting distributed generation (DG) sources closer to load demands in the distribution system (DS). These include cogeneration with high thermal efficiency, clean natural energy generation, and many others in recent years. DGs most especially the variable renewable generation (RG), impose new challenges to the DS over centralized predictable production due to their intermittency and fluctuating characteristics [1]. The integration of DGs into the DS, that is close to consumer point of common coupling (PCC), changes the traditional flow of power from a unidirectional flow (generation, transmission and distribution) to a bidirectional flow that may bring imbalance to energy production and consumption [2]. Critical operational factors in the distribution system could arise accordingly such as voltage, frequency, and reactive power control [2].

Therefore, it is required that the whole network operational system be revisited and managed in a more innovative way than with the conventional manner. In order to minimize the negative impacts brought forward by DGs on the DS and maximize associated benefits, technical constraints concerning the integration of DG units and their penetration levels are being gradually adopted. The optimal sizing and siting of DG units can be used to enhance reduction in power losses, power transfer capability, increase system power quality and reliability, and improve the voltage profile [3]. Incongruous installation of DGs at haphazard locations can result in a rise in system losses and poor voltage profile. Optimal sized DG units and types should be located appropriately to acquire system wide benefits.

With the trend to increase the penetration level of firm and variable DGs in distribution networks, a number of studies were conducted to investigate the criteria for optimal sizing and siting of various DGs.

\footnotetext{
* Manuscript received June 15, 2013; revised July 31, 2013.

Corresponding author Tel.:+60 755 57164; E-mail address: m.shaaban@fke.utm.my.
} 
Wills [4] employed the ' $2 / 3$ rule' to determine the appropriate bus for DG siting, that is, a DG unit and rating of $2 / 3$ of the load at $2 / 3$ of the feeder length, downstream from the source substation. However, this rule assumes uniformly distributed loads in a radial configuration. Rau [5] presented a power flow algorithm for optimal DG sizing at each load bus. The algorithm proposed, nonetheless, is not efficient due to the large number of load flow computations involved. Acharya et al. [6] proposed an analytical approach to minimize power losses through optimal sizing and placement of a DG. In addition, the authors suggested an approximate loss formula to determine the optimal DG units. Yet, the adopted DG unit injected only reactive power. In [7], an analytical approach is presented for the allocation of a DG in a distribution system. However, the method only finds the optimal placement of the DG, but could not determine the size. The previous algorithms, centering on power losses, are only suitable for traditional passive networks. Voltage issues could appear due to variation of demand and/or renewable generation. It is therefore necessary to consider the losses and voltage issues in an integrated perspective.

This paper presents a simple analytical technique to determine the optimal size and optimal siting of distributed generation in distribution systems for active power loss reduction and voltage profile enhancement. DG unit sizing and location are calculated using a sensitivity index that combines the exact loss formula and voltage sensitivity coefficients. Test results carried out on the IEEE 13 bus feeder are presented and analyzed.

\section{Optimal Sizing and Placement of DG}

Technical benefits of introducing DGs in the DS can be accrued categorically through the reduction in line losses and emissions, as well as improvement of power quality and voltage profile. According to the voltage variation across the feeder, there could be voltage rise/drop in the DS for any significant amount of power injected by the DG. Location and sizing issues of DGs, including methods of voltage regulation, are other factors affecting voltage variation. Furthermore, the size of the DS itself, as far as the load in MW is concerned, could be influential in DG sizing [6].

\subsection{Optimal placement of $D G$}

A sensitivity Index (SI) is proposed in this paper to determine the best location of the DG. The proposed index combines the exact loss formula [6] and voltage sensitivity coefficients. The sum of total power loss and voltage deviation due to DG injected power is contemplated:

$$
\begin{aligned}
S I_{i} & =\frac{\partial P_{L}}{\partial P_{i}}+\gamma_{i} \frac{\partial V_{i}}{\partial P_{i}} \\
P_{L} & =\sum_{i=1}^{N} \sum_{j=1}^{N}\left[\alpha_{i j}\left(P_{i} P_{j}+Q_{i} Q_{j}\right)+\beta_{i j}\left(Q_{i} P_{j}+P_{i} Q_{j}\right)\right]
\end{aligned}
$$

where

$$
\alpha_{i j}=\frac{r_{i j}}{v_{i} v_{j}} \cos \left(\delta_{i}-\delta_{j}\right), \beta_{i j}=\frac{r_{i j}}{v_{i} v_{j}} \sin \left(\delta_{i}-\delta_{j}\right),
$$

and $Z_{i j}=r_{i j}+x_{i j}$ are the $i j$ th element of $\left[Z_{\text {bus }}\right]$ matrix, $\gamma_{i}$ is the weighting factor, $P_{i}=P_{D G i}-P_{D i}$ $Q_{i}=Q_{D G i}-Q_{D i}, P_{i}$ and $Q_{i}$ are active and reactive power of the bus, $P_{D G i}$ and $Q_{D G i}$ are power injection of DGs to the bus, while $P_{D i}$ and $Q_{D i}$ are the loads at bus $i$. The use of the weighing factor $\gamma$ allows the emphasis on certain critical attributes of DG location relative to power losses or voltage variation. A weight value of unity indicates comparable emphasis of losses and voltage variations. Using the exact loss formula, the sensitivity of active power losses with respect to active power injection from the DG is given by [6]:

$$
\frac{\partial P_{L}}{\partial P_{i}}=\alpha_{i}=2 \alpha_{i i} P_{i}+2 \sum_{j=1, j \neq i}^{N}\left(\alpha_{i j} P_{j}-\beta_{i j} Q_{j}\right)
$$


The voltage sensitivity coefficient in (1) is used to link the variations of the bus voltages to the DG power injections. Each coefficient linearizes the dependency between the $i$ th bus injected power and the $i$ th bus voltage magnitude. A typical expression for the computation of these sensitivities, is the use of updated Jacobian matrix derived from the power flow equations [8]:

$$
\left[\begin{array}{c}
\Delta P \\
\Delta Q
\end{array}\right]=\left[\begin{array}{ll}
J_{P \theta} & J_{P V} \\
J_{Q \theta} & J_{Q V}
\end{array}\right]\left[\begin{array}{c}
\Delta \theta \\
\Delta V
\end{array}\right]
$$

Assuming unity power factor, then, $\Delta Q=0, J_{Q \theta} \Delta \theta+J_{Q \theta} \Delta V=0$, and $\Delta \theta=-J_{Q \theta}^{-1} J_{Q V} \Delta V$. Substituting for $\Delta \theta$ and expanding, yields

$$
\Delta P=\left[J_{P V}-J_{Q \theta}^{-1} J_{Q V} \Delta V\right] \Delta V
$$

Therefore,

$$
\frac{\Delta V}{\Delta P}=\left[J_{P V}-J_{Q \theta}^{-1} J_{Q V} \Delta V\right]^{-1}
$$

With the voltage sensitivity factors calculated, the sensitivity index (SI) can be evaluated for all buses. The bus with the highest SI corresponds to the best location of the DG.

\subsection{Optimal sizing of the $D G$}

The total active power losses of the system is a parabolic function that becomes minimum, if the partial derivatives of (2), with respect to active power injection from the DG at bus $i$, tend to zero. Therefore, and upon rearrangement and simplification, eq. (2) becomes:

$$
P_{i}=\frac{1}{\alpha_{i i}} \sum_{j=1 . j \neq i}^{N}\left(\alpha_{i j} P_{j}-\beta_{i j} Q_{j}\right)
$$

Therefore,

$$
P_{D G i}=P_{D i}-\frac{1}{\alpha_{i i}} \sum_{j=1, j \neq i}^{N}\left(\alpha_{i j} P_{j}-\beta_{i j} Q_{j}\right)
$$

Equation (7) gives the optimal size of the DG at bus $i$.

\section{Computational Procedure for Optimal Location of the DG}

The optimal siting of the DG is determined by the bus with the highest SI, whereas the optimal size is obtained from (7). The details of each step are described as follows:

(i) Run the base case power flow and find the Jacobian;

(ii) Calculate $\frac{\partial P_{L}}{\partial P_{i}}$ using (3) and calculate $\frac{\partial V_{i}}{\partial P_{i}}$ using (5);

(iii) Find the $S I$ as in (1), with appropriate weighting;

(iv) Calculate the optimal DG size from (7) at the bus determined in step (iii);

(v) Integrate the optimal DG size at the optimal location and run the power flow;

(vii) Integrate multiple DGs of the same size in step $v$ at different buses and run the power flow.

The above steps not only determine the appropriate size and site for the DG, but also conduct further verification through exhaustive search among network buses. The use of multiple DGs is adopted to better represent the characteristics of modern distribution networks.

\section{Test Results}

The proposed method is tested on a sample circuit taken from a modeled IEEE 13 test feeder of an

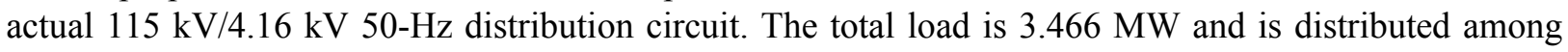


commercial and residential energy consumers. The IEEE 13 test feeder, shown in Fig. 1, consists of a three-phase overhead or underground primary feeders and double-phase or single-phase line sections near the end of the feeder laterals. Loads with different types including constant current, constant impedance and constant power are modeled at the system buses. The feeder is supposed to have a unity power factor. Therefore, the power injections of the DGs have been represented by voltage-independent active injections with zero reactive power. The optimal DG size of $2.5 \mathrm{MW}$, calculated according to (7) at bus 3, is integrated into the distribution feeder for the simulation work. Base case power flow was carried out on the feeder so as to calculate the loss sensitivity and the voltage sensitivity coefficients comprising the SI. The SI of the DG is calculated at each bus for the test system based on the analytic expression in (1). The data for the IEEE 13 Node Test feeder distribution network is taken from [9], with minor modifications of base values of $5 \mathrm{MVA}$ and $4.16 \mathrm{kV}$.

The calculated SI for each respective bus is shown in Fig. 2. It is clear that bus 7 has the highest $S I$ of 0.102584 . However, that bus is not suitable for the location of the DG, because the DG connected at bus 7 should be installed at $0.480 \mathrm{kV}$ while the DG is modeled at $4.16 \mathrm{kV}$. Therefore, the next subsequent bus with high sensitivity index, as illustrated in Fig. 2, is bus 9. In other words, bus 9 is the appropriate location of the DG.

Fig. 1. IEEE 13 bus test feeder.
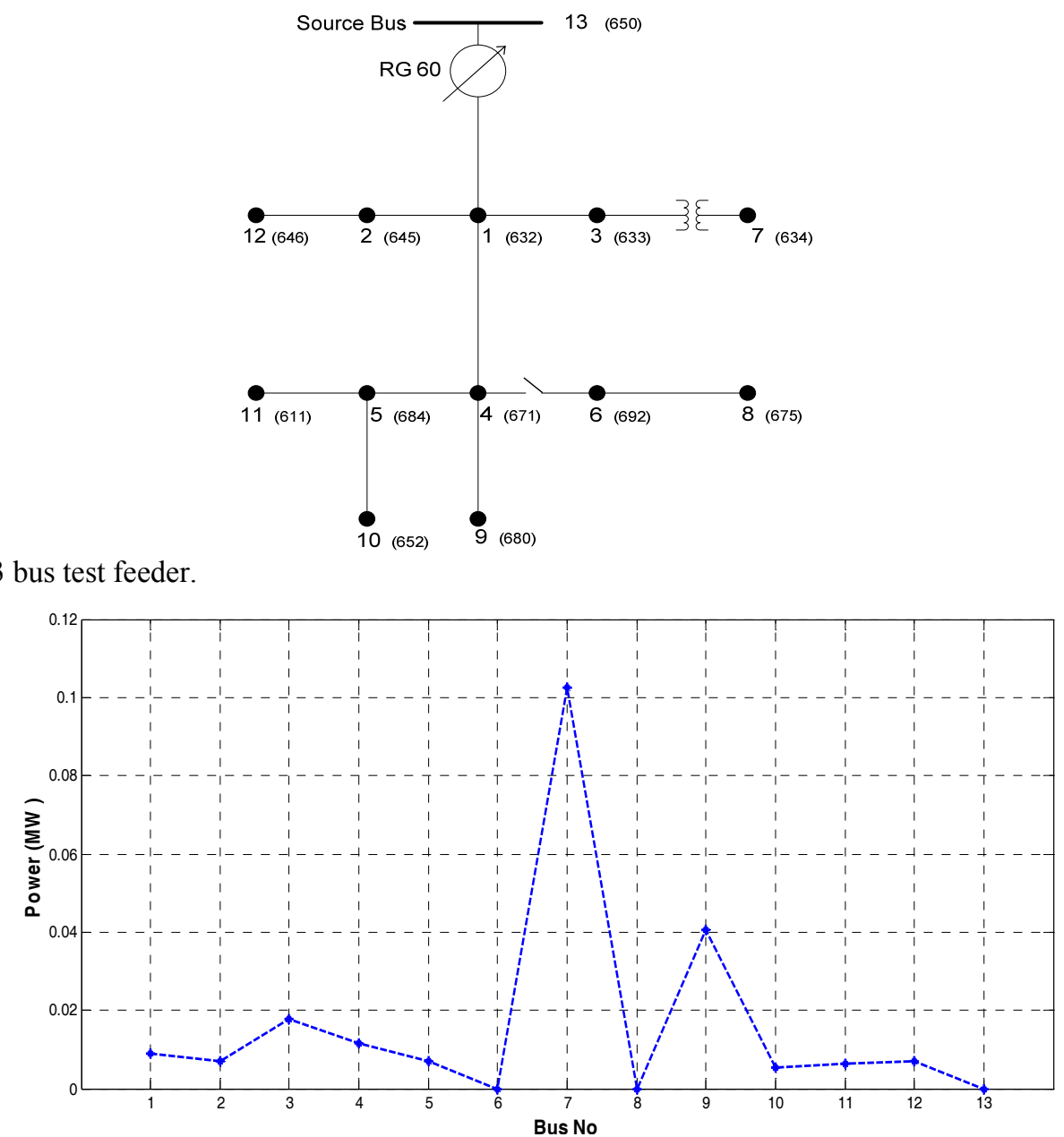

Fig. 2. Sensitivity index.

Case I: Comparison of voltage profile of DG at non-optimal and optimal location

A 2.5 MW DG is connected at an arbitrary location at bus 3 and the selected location at bus 9; one at a time. Power flow was carried out to get the voltage profiles. Fig. 3 shows the voltage profile without DG (base case), with DG at a non-optimized location (bus 3) and at optimal location (bus 9). It is observed that there is little or no improvement in the voltage profile with non-optimal siting of the DG at bus 3 , as 
compared with better improvement in the voltage profile with the optimal siting of the DG at bus 9 of the test feeder. This shows that bus 3 is not sensitive to DG, while bus 9 is sensitive to DG siting. The minimum voltage in bus 11 is improved from $0.961 \mathrm{pu}$ without DG to $0.976 \mathrm{pu}$ with DG at the optimal location. The integration of DG at the optimized location in the feeder sections reduce the system losses better than at a non-optimal location as shown in Fig. 4 and Table 1, which depicts the system losses along the feeder lines. The 2.5 MW DG is connected to each bus of the feeder, one at a time to determine the total power losses due to the connection of DG at such location. Fig. 5 demonstrates the total power losses as a result of the DG at each bus. Bus 9, the selected location of the DG has a total power loss of $50.9 \mathrm{~kW}$ as shown in Fig. 5, compared with other buses. It is evident from Fig. 5 that, buses 4, 6, and 8 render lower losses. However, they do not correspond to the lowest voltage deviations. This indicates the efficacy of the proposed $S I$ index in identifying the best siting locations.

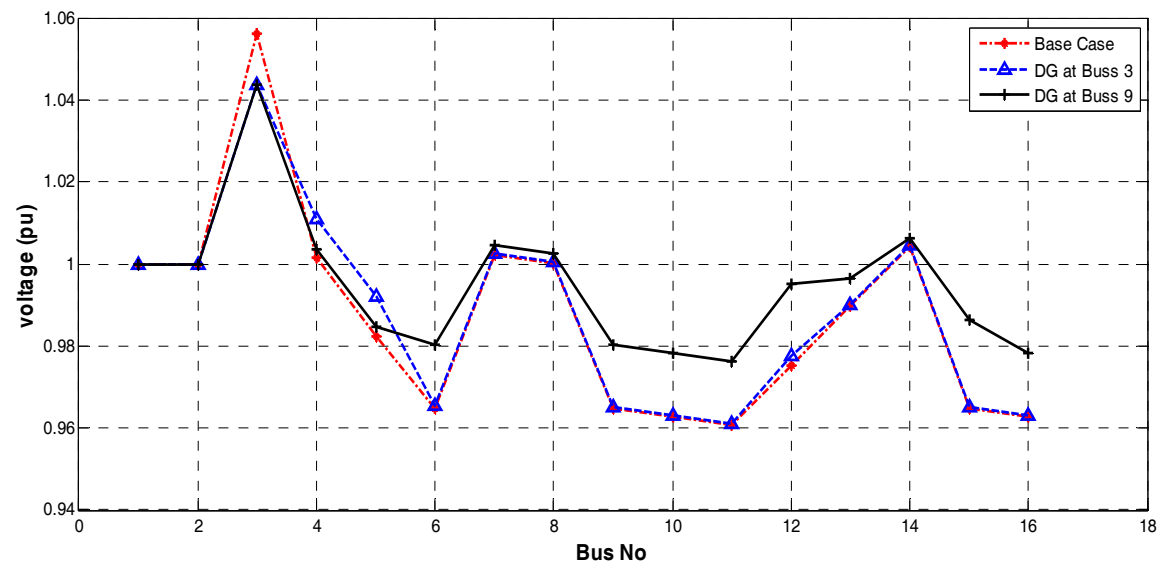

Fig. 3. Voltage magnitude (pu) at bus 3 and 9.

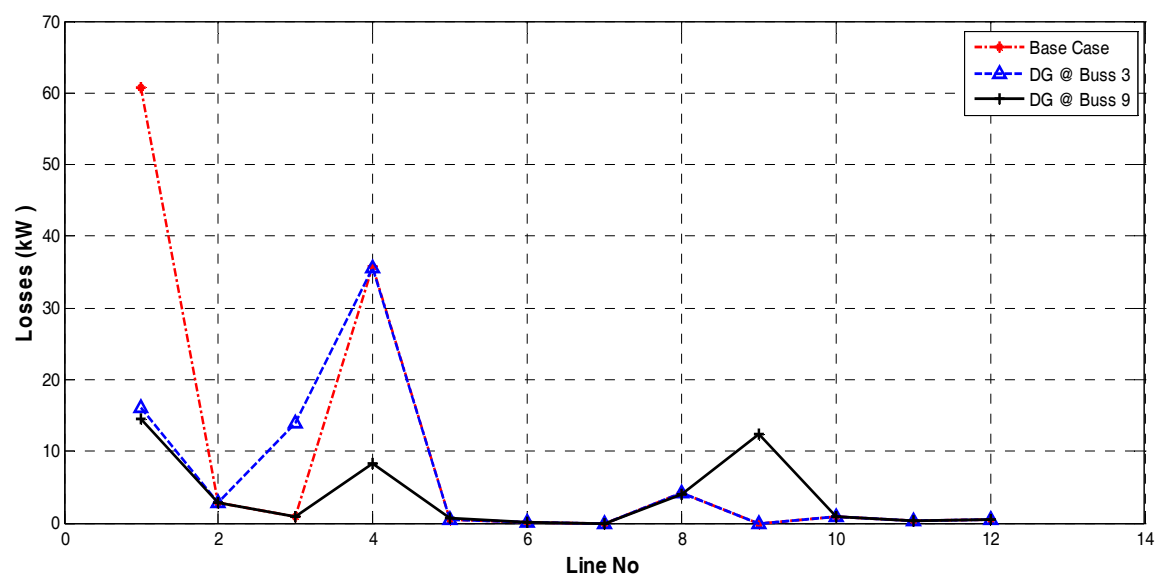

Fig. 4. System losses along the feeder lines.

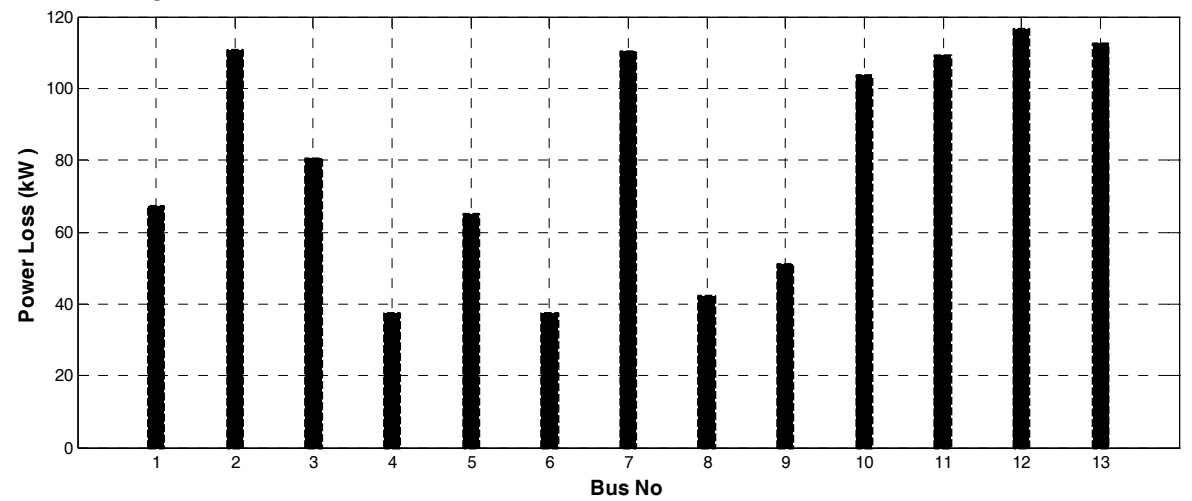

Fig. 5. System losses at each bus. 
Table 1. Summary of losses at different cases

\begin{tabular}{lcccc}
\hline \hline Case & Line losses $(\mathrm{kW})$ & Transformer losses $(\mathrm{kW})$ & Total losses $(\mathrm{kW})$ & Circuit losses (\%) \\
\hline Base Case & 106.5 & 5.9 & 112.4 & 3.25 \\
DG at 3 & 75.0 & 5.5 & 80.5 & 2.33 \\
DG at 9 & 45.3 & 5.6 & 50.9 & 1.47 \\
PV at 12 & 116.4 & 5.8 & 122.2 & 3.52 \\
PV \& ES at 12 & 95.3 & 5.8 & 101.2 & 2.92 \\
\hline \hline
\end{tabular}

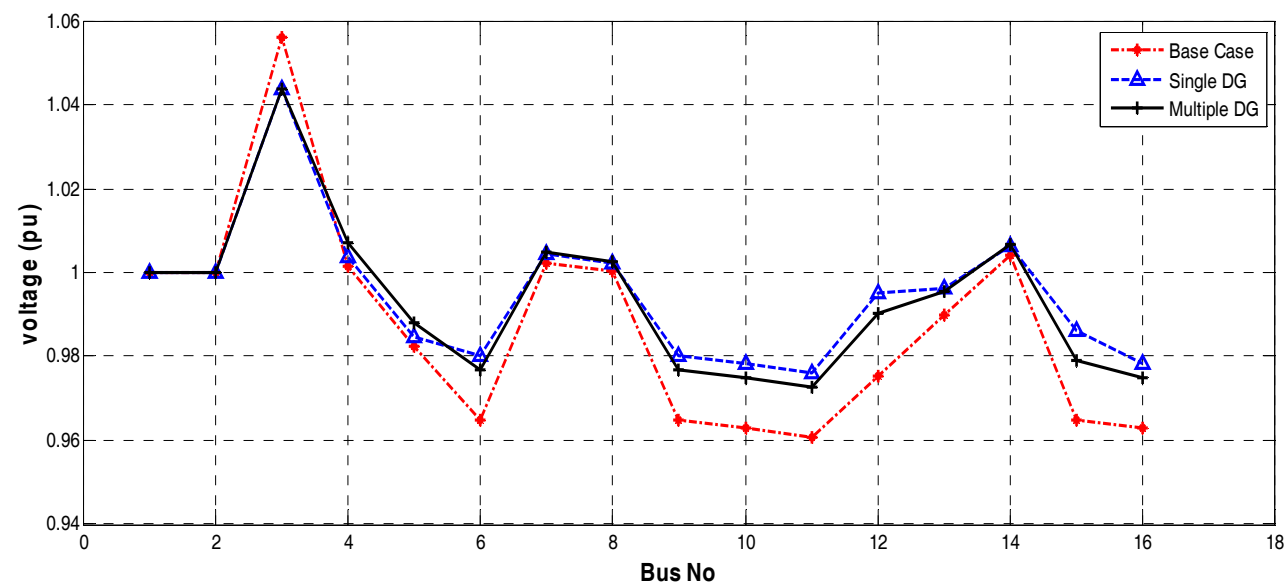

Fig. 6. Voltage magnitude of single and multiple DGs.

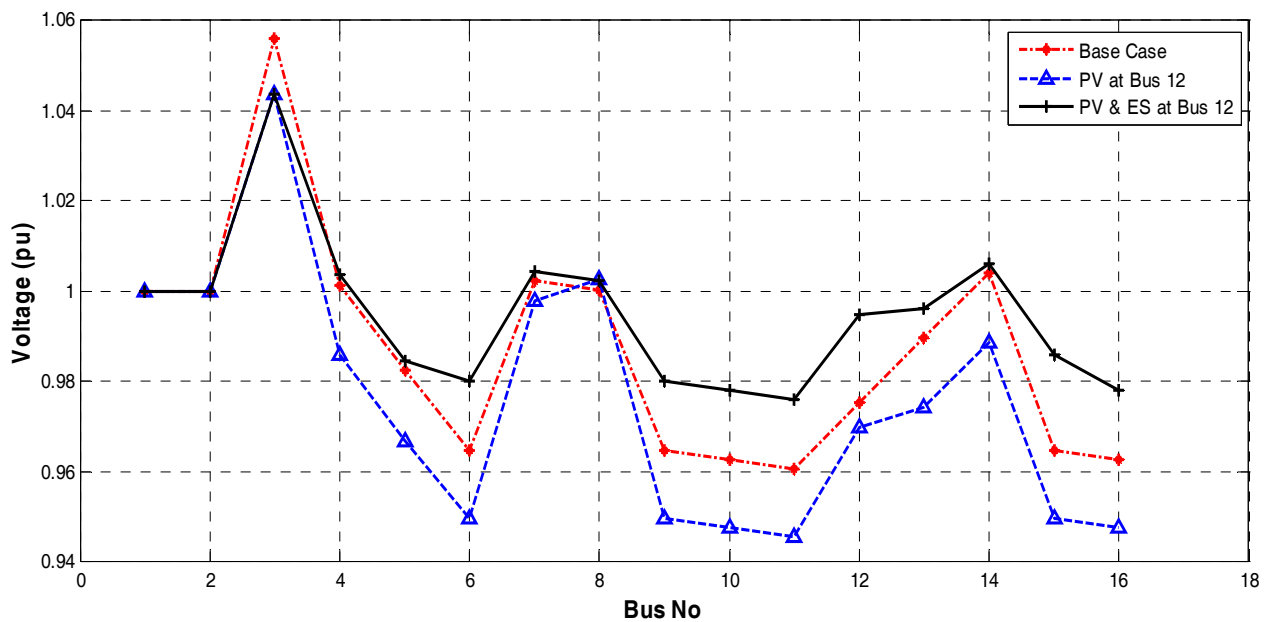

Fig. 7. Effect of renewable generation on voltage profile.

\section{Case II: Effect of multiple DG on voltage profile}

A total of 2.5 MW DG power was distributed among buses 3, 6 and 9 in order to appreciate the effect of multiple DGs on the distribution system. The result of the power flow in Fig. 6 depicts that; a single DG in an optimal location can improve system voltage profile and reduce system losses better than multiple DGs of the same combined capacity in different arbitrary locations.

Case III: Effect of non-dispatchable renewable energy on voltage profile

The variability of solar and wind energy, representing variable renewable generation, could have a significant impact on the voltage profile at the bus where they are connected. Both solar and wind are not always available where and when needed. They both have uncontrollable and non-dispatchable power output, resulting in intermittent generation. Therefore, the size and location cannot be determined a priori through any optimization criterion, since they largely depend on the amount of possible energy harvested at a particular time of the day and at a location dictated by the available weather conditions. It is observed in Fig. 7 that a $2.5 \mathrm{MW}$ PV connected at bus 12 (non-optimal location) based on the assumption of sunlight availability at that location and at a $50 \%$ capacity credit, resulted in degraded voltage profile and 
higher system losses as shown in Table 1. The voltages at six buses of the feeder are below the acceptable limit (0.95-1.05). For example, the voltage at bus 6 is $0.948 \mathrm{pu}$. Application of a smart grid technology such as energy storage could improve the voltage profile and enhance system performance. The voltage at bus 6 with a battery energy storage of $1.25 \mathrm{MW}$ connected at bus 12, alongside the PV, is increased to $0.957 \mathrm{pu}$; which lies within the acceptable limits.

This result does not only underline the importance of attaching energy storage, as an exemplar of smart grid technologies, with variable renewable generation such as solar energy to maintain acceptable system performance, but also brings to attention the deficiency of current analysis tools to capture the fickle inherent characteristics of variable DGs. A framework which accounts for the time-varying of characteristics of renewable DGs to minimize the energy losses, rather than the power losses, and enhances the voltage profile, in a given time horizon, is needed.

\section{Conclusions}

The sizing and siting of DG is a crucial element in the planning and operation of a distribution system. The optimal sizing and placement of DG units can be used to reduce power losses, increase system reliability and improve overall voltage profile. This maintains the voltage magnitude at the customer terminal at nominal values and reduces losses in the feeder. The optimal siting of DG was achieved via a simple analytic sensitivity index that combines power loss and voltage variations through appropriate weighting, whereas the size of the DG is obtained through power loss sensitivity. Results from examining the IEEE 13 test feeder showed that the system power loss is successfully reduced and the voltage profile is improved with the proposed method. The paper has also highlighted the inherent challenges associated with the integration of intermittent renewable generation into the distribution network, where the size and location are ordained by merits of resource availability and appropriate metrological conditions. The use of smart grid technologies such as energy storage, as illustrated in the paper, can effectively assist to harness non-dispatchable renewable energy resources and bring further improvement to overall voltage profile and total system losses. It is clear that several other aspects of DG require further analysis, which provides a fertile ground for future studies.

\section{References}

[1] Aghaei J, Alizadeh MI. Demand response in smart electricity grids equipped with renewable energy sources: A Review. Renewable and Sustainable Energy Reviews, Feb.2013; 18:64-72.

[2] Carvalho PMS, Correia PF, Ferreira L. Distributed reactive power generation control for voltage rise mitigation in distribution networks. IEEE Transactions on Power Systems, 2008. 23(2):766-772.

[3] Petinrin OJ, Shaaban M. Overcoming challenges of renewable energy on future smart grid. TELKOMNIKA Indonesian Journal of Electrical Engineering, 2012. 10(2):229-234.

[4] Willis HL. Analytical methods and rules of thumb for modeling DG-distribution interaction. Presented at: IEEE Power Engineering Society Summer Meeting, 2000.

[5] Rau NS, Wan Y-H. Optimum location of resources in distributed planning. IEEE Transactions on Power Systems, 1994. 9(4): 2014-2020.

[6] Acharya N, Mahat P, Mithulananthan N. An analytical approach for DG allocation in primary distribution network. International Journal of Electrical Power \& Energy Systems, 2006. 28(10):669-678.

[7] Wang C, Nehrir MH. Analytical approaches for optimal placement of distributed generation sources in power systems. IEEE Transactions on Power Systems, 2004. 19(4):2068-2076.

[8] Wood AJ, Wollenberg BF. Power Generation, Operation, and Control. 2nd ed. Wiley-Interscience; 1996.

[9] Sekar A, Mwakabuta N. Determination of feeder losses by an improved linear model in a radial circuit. Presented at: IEEE 39th Southeastern Symposium System Theory (SSST'07), 2007. 